\title{
NANOSTRUCTURED LIPID CARRIERS: THE FRONTIERS IN DRUG DELIVERY
}

\author{
KARNATI V CHANDANA*, N. VISHAL GUPTA, SANDEEP KANNA
}

Department of Pharmaceutics, JSS College of Pharmacy, JSS Academy of Higher Education and Research, Sri Shivarathreeshwara Nagara, Mysuru, Karnataka, India. Email: chandanakarnati3@gmail.com

Received: 12 April 2019, Revised and Accepted: 14 May 2019

ABSTRACT

By the modification of solid-lipid nanoparticles (SLNs), the nano-structured lipid carriers (NLCs) were obtained by the modification of nanostructured that hold the qualities of the SLN, increases the stability of the drug, and prevents drug leakage. The limitations of the drugs that are delivered in the body using different routes are poor solvency, first pass metabolism, and poor bioavailability hence to overcome these limitations NLCs were prepared. Oral, topical, transdermal, ocular, and parenteral are the different routes through which nanobased systems are delivered. Spatially incompatible liquid lipids and solid lipids are blended and NLCs were prepared. It stays solid at room temperature. The advantages of for drug therapy over conventional carriers NLCs are increased solubility, improved bioavailability. This review describes about the NLC, its advantages and disadvantages, types, NLC techniques, mode of action, and applications of NLC. Imperfect, amorphous, and multiple types are the main three types of NLCs. Lipids, liquid lipids, solid lipids, Emulsifiers, ultraviolet (UV) blockers, and aqueous medium were the excipients used. Microemulsion technique, microemulsion based SLN/NLC preparations, solvent diffusion method, high-pressure homogenization (HPH), hot and cold homogenization techniques, and melt emulsification method are the techniques used for the preparation of NLC. The applications of NLCs are for ocular, brain targeting, topical, cancer therapy, cosmetics, and analgesia.

Keywords: Nano structured lipid carriers, Solid lipid nanoparticles, Solid lipids, Liquid lipids.

(C) 2019 The Authors. Published by Innovare Academic Sciences Pvt Ltd. This is an open access article under the CC BY license (http://creativecommons. org/licenses/by/4. 0/) DOI: http://dx.doi.org/10.22159/ajpcr.2019.v12i7.33595

\section{INTRODUCTION}

Nanostructures are the particles which range from 10 to $1000 \mathrm{~nm}$, by which the drug molecules are absorbed or attached which are dissolved, entrapped. NLCs have efficient properties. NLCs are the second generation of lipid nanocarriers and are made up of matrix which contains solid lipid and liquid lipid. To overcome the disadvantages of SLN, NLCs have been developed. The nanotechnology has been widely used in diagnostics, tissue regeneration, and cell culture. The traditional colloidal systems have such limitations and nanoprobes, liposomes, and nanopores [1-4].

Speiser et al. prepared the lipid nano pellets of the first generation for oral administration and divided into nanocarriers and nanodrugs. By the dissolution or dispersion of drugs, nanocarriers are prepared, which may be classified as either nanospheres or nanocapsules. Polymers and lipid materials are the materials used. Combination of solid and liquid lipids and SLNs were modified as the delivery systems synthesized by cooling and heating crystallization. The carriers used for the NLCs are biodegradable and exhibits low in vivo toxicity. Drug loading properties exhibited by NLC, hydrophobic, or hydrophilic drugs NLC, hydrophobic, or hydrophilic drugs. Certain organizational targeting pattern is exhibited by the carrier [5]. NLC particles are stable in their physical and chemical properties. For topical, dermal, transdermal administration, and NLCs were successfully utilized. Solid and liquid lipids are mixed and are used for the preparation of aqueous dispersions of solid nanoparticles, and stabilized by one or two surfactants. To improve skin hydration, NLCs are extensively utilized [5-8].

NLC facilitates loading capacity for drug accommodation and it contains different $\mathrm{C}$-chains in the crystalline structure. Ascorbic palmitate, clotrimazole, ketoconazole, and other antifungal agents' show controlled release behavior. By replacing liquid oil with solid lipid and lipid nanoparticles, the solid matrix has been formed. The origin of SLNs was created at the start of 1990. Usage of physiological lipids is the major advantage of SLNs. Low incorporation due to the crystalline structure of solid lipids is the major disadvantage of SLNs as drug carriers. To overcome the problems raised by SLNs, NLCs were developed [9-12].

\section{BENEFITS OF NLCS}

- Biodegradability, greater drug protection is the advantages which were exhibited by the NLCs

- $\quad$ NLCs were used as the extended release of the drug

- NLCs were used for the entrapment of hydrophobic drugs and hydrophilic drugs

- Due to the lesser size of lipid particles, the drug penetration into the mucosa is increased

- $\quad$ NLCs considered as safe.

\section{LIMITATIONS OF NLCS}

- Cytotoxic effects are observed because of the nature of matrix and concentration

- $\quad$ Some surfactants exhibit irritative and sensitizing actions

- Clinical and preclinical studies lacked in the preparation of NLCs.

\section{TYPES OF NLCS}

NLCs are classified into three types:

1. The imperfect type

2. The amorphous type

3. The multiple type.

\section{The imperfect type}

The imperfections are formed by blending solid and liquid lipids chemically and increase drug loading. Liquid phase lipid (oil) is present in the minute amounts in the imperfect type of NLC. During the crystallization process of production, there will be expulsion of drugs in the imperfect NLC [13-16].

\section{The amorphous type}

NLCs are obtained by blending special types of solid and liquid lipids in a controlled manner (e.g., isopropyl myristate). This type of NLC forms solid lipid that lacks any crystalline structure [17-19]. 


\section{The multiple type}

This contains solid lipid encapsulates oil nano compartments. In the oil compartments, the drug is loaded/loaded. Lipid-lipid precipitation method was used for the preparation. In the lipid matrix, high concentration of liquid lipids is mixed and the drug expulsion can be avoided by the multiple types of NLC. Oil reaches its solubility limit and precipitates into nano compartments during the cooling effect [20-23].

\section{EXCIPIENTS USED IN NLCS}

\section{Lipids}

The inner cores consist of both solid and liquid lipids. Glyceryl behenate, glyceryl palmitostearate, fatty acids, triglycerides, steroids, and waxes were the solid lipids frequently used for NLCs. At room temperature, these lipids are in the solid state. The lipids melt at high temperatures. Digestible oils from natural sources were used in NLC. Liquid lipids consist of Miglyol 812, which is a medium chain triglyceride (MCT) which has similar structures to Compritol ${ }^{\circledR}$ used as a constituent. 2-octyl dodecanol, paraffin oil, isopropyl myristate, propylene glycol dicaprylocaprate, and squalene are also used. Oleic acid, linoleic acid, and decanoic acid, which are penetration enhancers were also used for topical delivery. A - tocopherol and other tocols were used for nanoemulsions. Due to their stability and good solubility in lipophilic drugs, tocols are very effective oils. The currently popular NLCs are produced by natural oils from plants.

\section{Liquid lipids}

Digestible oils derived from natural sources are the most frequently used liquid lipids for NLCs. MCT and oleic acid are the famous liquid lipids. To fabricate NLCs, the liquid lipids such as edible oils such as soya bean oil and sunflower oil can be used. Among the natural edible oils, oleic acid is a common component. Using hydrolysis, oleic acid can be prepared from vegetable oils. In neutraceutical formulation, oleic acid which was used as an emulsifier has low viscosity. By esterification and fractionation methods MCT which is food graded obtained. By the USFDA, MCT was recognized as safe and has high stability against oxidation and an emulsifying agent. The smell of the foods has been influenced by the free fatty acids which are obtained by the hydrolysis of MCT. These oils have been utilized as antioxidants which protects nourishment from oxidation; some oils incorporate $\gamma$-tocopherol, generally appeared in corn oil. Likewise, they are economical contrasted with oleic acid and MCT. They have more level of unsaturation and consistency, so they are unacceptable to encapsulate some hydrophobic food actives. Low encapsulation effectiveness appeared by the soya bean oil contrasted with MCT and oleic acid, for example, paraffin oil[24-29].

\section{Solid lipids}

The most ordinarily utilized for manufacture of NLC is stearic acid, glyceryl monostearate (GMS), and glyceryl palmitostearate, and glyceryl behenate. They are especially surface active and food active compound carriers.

The essential part of vegetable and animal lipids, as a noteworthy component of hydrogenated fats, is stearic acid, which is an endogenous long-chain saturated fatty acid. It has been accounted for that stearic acid is biocompatible with human tissues and body fluid. Contrasted with its synthesized counterparts stearic acid has moderately lower toxicity and better biocompatibility (Fundaro et al., 2000). In the field of nutraceuticals, GMS which is a non-poisonous and non-irritating which has at the very least $40 \%$ of monoacylglycerol saturated fatty acids is broadly utilized. GMS can go about as a non-ionic emulsifying agent and plasticizer (Tamjidi et al., 2013). Stearic unsaturated fats and mono-, di-, and triacylglycerols of palmitate are the constituents which are available in glyceryl palmitostearate which has appeared promising sustained release profiles. Mono-, di-, and triacylglycerols of behenic acid are the segments which are available in glyceryl behenate. The high entrapment efficiency is shown by the glyceryl behenate solid lipids and promising stability due to numerous defects in the crystalline cross-section [30-33].

\section{Emulsifiers}

To stabilize the lipid dispersions, the emulsifiers have been widely used. Pluronic F68 (poloxamer 188) and polysorbates (Tween) were identified as the most widely used hydrophilic emulsifiers. For fabrication of NLCs, lipophilic emulsifiers such as Span 80 and lecithin are widely utilized. The particle aggregation can be prevented by the combination of emulsifiers which are used more efficiently. The nanoparticles shell has polyethylene glycol (PEG) prevents take up by the reticuloendothelial framework and to prolong the circulation time of drugs. The physical stability of lipid dispersions can be impaired by the preservatives. Hydrolite ${ }^{\circledR}$ five is proved suitable for the preservation of coenzyme Q10-loaded NLCs that has been demonstrated by Obeidat et al [34-36].

\section{UV blockers}

UV blockers were used to protect skin from UV radiation of the sun and it lowers the risk of skin cancer. The UV light has been absorbed by some sunscreen products which contains organic chemical compound, for example, Avobenzone absorb UV-A radiation [37-41].

\section{Aqueous medium}

The purified water has been used for the NLCs by reverse osmosis.

\section{NLC TECHNIQUES}

\section{HPH}

In large-scale production of NLCs, HPH has been extensively used. By the application of high pressure through very high shear stress on the lipid is applied and it is pushed, resulting in destruction of particles down to the submicrometer or nanometer size. $5 \%-10 \%$ is the range of the lipid contents usually. Scaling up problem has not been shown by the HPH. At both elevated temperature or below room temperature, homogenization may be performed [42-44].

\section{Cold homogenization method}

Cold homogenization includes high-pressure processing of a suspension. In the lipid stage first, the food active substances are broken up and scattered, followed by a quick cooling procedure, typically utilizing liquid nitrogen. By utilizing ball processing or other processing techniques, the acquired lipid matrix is milled and the particles are obtained. The temperature is essential to process the milling step should not surpass the temperature of the lipid melting point. In a cold emulsifying agent solution, the got particles are scattered and homogenized to create wellstabilized NLCs. From thermal degradation, this strategy protects food active compounds and improves their entrapment efficiency. Due to the high cooling rates, the compounds are uniformly distributed within the lipid matrix. Crystallization step can be controlled in this technique, and this is one of the major advantages. The desired crystal structure of NLCs can be obtained by the rapid cooling process. The larger particle size and wider size distribution of NLCs can be achieved by this technique compared to other techniques (Mehnert and Mäder, 2001).

\section{Hot homogenization technique}

The drug along with melted lipid is dispersed under constant stirring by a high shear device in the aqueous surfactant solution of the same temperature. Using a piston-gap homogenizer, the preemulsion was obtained and nanoemulsion is cooled down to room temperature where the lipid recrystallizes and nanoparticles are formed [45-48].

\section{Microemulsion technique}

In molten lipid, the drug is incorporated. The lipid melt was prepared by adding a mixture of water, cosurfactant(s), and the surfactant and is heated up to the same temperature as the lipids. The substances are mixed in the correct ratios for microemulsion formation and they are transparent, thermodynamically stable. Microemulsion was taken into consideration to obtain the particles of the required size. In a cold aqueous medium, the microemulsion was dispersed under mild mechanical mixing of hot microemulsion with water in a ratio in the range 1:25-1:50. Rapid recrystallization of the oil droplets occurs by adding the dispersion in cold aqueous medium [49-51]. 
Microemulsion-based SLN/NLC preparations

Gasco21 demonstrated, depends on the dilution of microemulsions SLN preparation techniques were obtained in the scientific community there are various definitions about the structure and dynamics of microemulsion. Microemulsions appear as bi-phasic systems composed of an inner and outer phase has been demonstrated by Gasco and other scientists. A transparent mixture was prepared using low-melting fatty acid, emulsifier, coemulsifiers, and water and stirred at $65^{\circ} \mathrm{C}-7^{\circ} \mathrm{C}$ and microemulsions were formed. In cold water under stirring the obtained, hot microemulsions were dispersed. 1:25-1:50 are the ratios of hot microemulsion to cold water. By the composition of the microemulsion, the dilution process was critically determined. Energy is not required to get a submicron particle size because the droplet structure is already present in the microemulsion. To determine the quality of the final lipid nanosuspension, temperature gradient and the $\mathrm{pH}$ values are the main parameters. Rapid lipid crystallization and prevention of aggregation are facilitated by high-temperature gradients. Due to the dilution step, the content of lipids is lower compared with the HPH-based formulations [52-54].

\section{Solvent-diffusion method}

The organic stage comprises the LM, lipophilic surfactant and medication which are broken down in organic solvents at raised temperature are the fundamental two stages. At room temperature $\left(25^{\circ} \mathrm{C}\right)$, the resultant organic solution was scattered in the aqueous surfactant under high mechanical agitation for a period of time to get NLC. In vacuum desiccators, the resulted dispersion was placed for $24 \mathrm{~h}$ to evaporate the residual organic solvent [55-57].

\section{Melt emulsification method}

Solid-liquid lipids are warmed and blended in this technique. To frame an organic phase, the medications are added to the previously mentioned blend. A coarse emulsion was resulted after adding the organic phase to the water phase, which contains a surfactant. To frame the NLCs HPH is applied. Absence of organic solvent residue, absence of burst discharge for the first time, and scatterings with high lipid concentration are the primary points of interest in this technique. This technique is not totally reasonable for industrial production, and there is residual organic solvent the main disadvantages in this method. This method was utilized by Zhiqiang Tian et al. with Precirol ATO 5 as the solid lipid and Captex 100 as the liquid lipid to entrap fenofibrate, yielding an encapsulation efficiency of $8.5 \%$ and particle size of $227.5 \mathrm{~nm}$ [58-60].

\section{MODULATION OF DRUG RELEASE}

By the mechanism of diffusion and simultaneously by lipid molecule degradation in the body, the medication is discharged from the lipids. At times, it may be attractive to have a controlled quick discharge beyond diffusion and degradation. When the particles are administered, the release should be triggered by an impulse. Due to their improper lipid structures, the NLCs accommodate the drug. To convert in a more ordered structure, the trigger impulse has to be applied to the matrix so that a desired burst drug release can be initiated. When the cream is applied to the skin, NLCs of certain structure can be triggered this way. The drug release can be done by an increase in temperature and water evaporation. Psoriasis can be treated by the cyclosporine-lipid particles. Itself the cream is saturated with cyclosporine, just as a cyclosporine-loaded NLC contained in the cream. The discharge from the lipid particles should lead to a supersaturated framework leading to improved penetration of cyclosporine into the skin after the application to the skin [61].

\section{APPLICATIONS}

\section{NLCs for cancer therapy}

Anticancer drugs can be incorporated into the NLCs. The improvement in chemical stability and cytotoxicity can be seen by incorporating anticancer drugs into the NLCs. In the melanoma and leukemia conditions, the NLCs that contains drugs such as camptothecin and topotecan drugs which are incorporated in NLCs fight against the cell in that condition and exhibits greater cytotoxicity and cell uptake. In lung cancer, ovarian cancer, breast cancer, and the docetaxel drug show antitumor activity. A strong cytotoxicity effect was shown by Duopafei ${ }^{\circledR}$, NLCs against cancer cells in the lungs. Compared to Duopafei, VEGFR2-antibody modified NLCs incorporated by docetaxel shows high cytotoxicity and great tolerance in mice against melanoma. Cytotoxicity was exhibited by VEGFR-2-antibody modified NLC in melanoma. To overcome multidrug resistance, the NLCs-dextran sulfate has been developed by Zhang et al.

Paclitaxel (PTX) and indocyanine green (ICG) for laser-triggered synergetic therapy of cancer

In chemo and photodynamic therapy, NLCs along with PTX was designed, which has more biocompatibility and tumor target ability. Under laser irritation, to increase the drug release, ICG was used, and it also acts as an imaging reagent. In the tumors bearing mice, NLC exhibited great tumor target ability. GMS, oleic acid, folic acid, Hoechst 33342, ICG, 2', $7^{\prime}$-dichlorfluorescein-diacetate, methylthiazoletetrazolium, 1,3-diphenylisobenzofuran, Stearic acid - PEG, and stearic acid-PEGfolate were the materials used in the preparation of NLC based PTX. For the protection and delivery of drugs to the tumor cells, the NLCs were used. Hence, tumor targeted codelivery of PTX and ICG mediated by NLC could be a effective strategy for cancer therapy [62-64].

\section{NLCs for topical drug delivery}

In the skin, there will be effective drug targeting and also increase in skin absorption by the NLCs. The acne lesion count and sebum secretion rate were decreased by cyproterone acetate. In the anti-acne therapy, the mechanism involved was the nanoparticles carry the drug into the follicles for efficient effect. The drug absorption in the human skin was enhanced 2-3 times by the incorporation of NLCs. In the effective treatment of psoriasis, acitretin was used. Compritol 255 and $45 \mathrm{mg}$ of Miglyol has been used for the preparation of NLC formulation and was the most promising formulation. NLC was used for drug penetration into the skin. Psoriasis can be treated by the combination of calcipotriol and methotrexate. Quercetin along with NLCs was used as an antiinflammatory agent and also used for the permeation of the drug and to increase the antioxidation exhibited by the quercetin. In conclusion, NLCs could be a promising vehicle for topical delivery of QT. In the major tropical diseases like gout, sunburn, rheumatoid arthritis the NLCs which incorporates the drug Flurbiprofen which is a NSAID used for treatment. In the major skin diseases related with inflammation like atopic dermatitis and psoriasis. Rapid onset and intermediate action are the properties exhibited by lidocaine categorized as a local anesthetic. Hydrogels of lidocaine loaded NLCs were used for topical applications [65].

\section{NLCs for brain targeting}

For Alzheimer's disease and other age-related diseases curcuminoid loaded NLCs were used for effective treatment. For the brain delivery of valproic acid through intranasal administration, rat electroshock model was used for the studies. Cetyl palmitate as the lipid matrix, squalene as the cationic surfactant, and Pluronic F68, polysorbate 80, and PEG as the interfacial additives used for the preparation of NLCs. For comparison, SLNs and lipid emulsions were prepared. Apomorphine which was used as an anti-Parkinson drug, was used as the model drug. Baicalein incorporated with NLCs given as intravenous injection used as a medication for the brain. Tripalmitin, Gelucires, Vitamin E, phospholipids, and poloxamer 188 were used in the NLC system. For the promotion of bromocriptine brain targeting, the carriers which were used are pluronic F68. In the disease such as Parkinson's disease and neuroleptic disease, the drug bromocriptine was used. For the treatment of Parkinson's disease, the apomorphine which is a dopamine receptor was used. The encapsulation percentage was $60 \%$ of apomorphine in the NLC. The anti-inflammatory and antioxidant effect was shown by biacalein protects against ischemic injury. In the brain, the NLC shows 7.5 folds increase compared to control. 


\section{Ocular delivery}

Chitosan Oligosaccharides (COS)-coated NLC for ocular drug delivery. By the method melt-ultrasonic, the flurbiprofen-NLCs were prepared and then coated with COS with a molecular weight of 3000$6000 \mathrm{kDa}$. Thiolated NLC was prepared by the conjugate of cysteinePEG monostearate and used as nanocarrier for the topical ocular administration of cyclosporine A. Acyclovir used as NLC was used for the faster permeation through the cornea, which indicates the increased corneal penetration properties. For the effective treatment of inflammatory, edematous, and in angiogenic ocular disease, the drug triamcinolone acetonide which is a corticosteroid was used. The drug which is incorporated in NLCs shows high bioavailability. By corneal and noncorneal pathways, the drug triamcinolone can be delivered effectively. For the anti-inflammatory ocular therapy, the drug Flurbiprofen-loaded NLCs were used which has stearic acid which was used as solid lipid.

\section{NLCs for cosmetics}

Cutanova nano repair Q10 cream was introduced in October 2005 used for cosmetic formulation and was compared with $\mathrm{o} / \mathrm{w}$ cream without NLC with respective to particle size, melting behavior, rheological properties. Cutanova nano repair Q10 cream was tested for the epicutaneous patch which has no irritating effects on the skin. For the treatment of inflammation, a topical gel of Valdecoxib-NLC was prepared for faster and yet prolonged action for the inflammation. NLCs prepared by microemulsion template technique. To improve the safety profile and to decrease the side effects, Fluticasone propionateNLC was prepared. Glyceryl palmitostearate, and PEG-containing MCT mixture, stabilized by polysorbate 80 , and soybean phosphatidylcholine were used for the preparation. For the blockage of UV radiation, NLCs which has sunscreen effect was used. Dr. Kurt Richter GmbH launched the first NLCs for cosmetic application, namely Nano Lipid Restore $\mathrm{CLR}^{\circledR}$ in 2006 . For the protection of skin from photodamage, the lutein which is used as a topical delivery was used effectively due to its antioxidant and antistress. For the testing of percutaneous absorption and photostability, the octyl-methoxy cinnamate loaded in NLC was discovered by Puglia et al. Blue light filter and UVB absorption are the characteristics of Octyl-methoxycinnamate. When the carnauba wax contains lipophilic filters, the SPF value was achieved higher than $45 \%$ compared with beeswax NLCs [65].

\section{Analgesia}

In the treatment of chronic pain and opioid dependence, the drug buprenorphine was used. In the liver and gut, the drug undergoes extensive first-pass metabolism. Hence, it is formulated as parental injection and as a sublingual tablet. $2.75 \mathrm{~h}$ is the half-life of buprenorphine. In the formulation of NLCs loaded buprenorphine, linseed oil and cetyl palmitate were used as liquid, solid lipids by Wang et al. 180-200 $\mathrm{nm}$ is the range of the diameter of nanoparticles. For the sustained release of NLCs, the ester prodrugs of buprenorphine with different alkyl chains were used. The extension of analgesic latency up to $10 \mathrm{~h}$ was shown by buprenorphine in tail-flick test in rats through intravenous. There is minimal toxicity of nanocarriers by the demonstration of erythrocyte hemolysis. For efficient analgesia, the combination of prodrugs and NLCs was used [66-68].

\section{CONCLUSION}

By combining a large portion of benefits of different novel colloidal delivery systems and by avoiding some of their limitations, NLCs are prepared, which are biodegradable, biocompatible transporter system. The types and concentrations of lipids and emulsifiers used to manufacture NLCs have to influence on loading capacity, entrapment efficiency, discharge characteristics, and physicochemical stability. Flexibility in medication loading, modulation of release, and improved performance in producing final dosage forms such as creams, tablets, and injectable are the main advantages of NLC. To treat different infections by the alternative routes, the NLCs ought to be kept on broadening their applications. Highly concentrated lipid particle dispersions are created in parallel to the NLC technology appropriate to both NLC and SLN, which facilitates the change of aqueous dispersions to solid products. At the opportune time, at the right center, the NLCs are the drug delivery system that reaches the targeted site in the body. The point has been to make therapeutic nanotechnology undertaking, especially for targeted drug therapy. As these delivery systems can be successfully scaled up for large scale manufacturing, hence, has a huge amount of potential from an extremely prolonged stretch of time. Camptothecin and topotecan drugs are used against cancer therapy. Docetaxel shows tumor activity. Duopafei was used against lung cancer. PTX and ICG for laser synergistic therapy of cancer. Cyproterone acetate was used to decrease sebum secretion and acne lesion count. For the treatment of psoriasis, acitretin was used. Methotrexate and calcipotriol combination was used against psoriasis. Curcuminoid loaded NLCs were used against Alzheimer's disease. Apomorphine was used as an anti-Parkinson drug. For the brain targeted diseases Biacalein was used. Lutein was used as topical delivery in cosmetics. Buprenorphine was used against chronic pain and opioid dependence.

\section{ACKNOWLEDGMENT}

The author expresses the gratitude to the JSS Academy of Higher Education and Research and JSS College of Pharmacy, Mysuru, for providing necessary support in due course of the work.

\section{AUTHOR'S CONTRIBUTIONS}

All the authors have contributed equally in the design, development, review, and finalization of the contents of the manuscript.

\section{CONFLICTS OF INTEREST}

The authors confirm that this article content has no conflicts of interest.

\section{REFERENCES}

1. Wang JJ, Liu KS, Sung KC, Tsai CY, Fang JY. Lipid nanoparticles with different oil/fatty ester ratios as carriers of buprenorphine and its prodrugs for injection. Eur J Pharm Sci 2009;38:138-46.

2. Mitri K, Shegokar R, Gohla S, Anselmi C, Müller RH. Lipid nanocarriers for dermal delivery of lutein: Preparation, characterization, stability and performance. Int J Pharm 2011;414:267-75

3. Venkateswarlu V, Manjunath K. Preparation, characterization and in vitro release kinetics of clozapine solid lipid nanoparticles. J Control Release 2004;95:627-38.

4. Sezer AD. Recent Advances in Novel Drug Carrier Systems. Rijeka, Croatia: InTech; 2012.

5. Pradhan M, Singh D, Singh MR. Novel colloidal carriers for psoriasis: Current issues, mechanistic insight and novel delivery approaches. J Control Release 2013;170:380-95.

6. Achieve HT. Characterizing release from lipid-based formulations. In: Hauss D, editor. Oral Lipid-Based Formulations: Enhancing the Bioavailability of Poorly Water Soluble Drugs. Boca Raton: CRC Press; 2007. p. 241.

7. Augustin MA, Sanguansri L, Lockett T. Nano- and micro-encapsulated systems for enhancing the delivery of resveratrol. Ann N Y Acad Sci 2013;1290:107-12.

8. Babazadeh A, Ghanbarzadeh B, Hamishehkar H. Novel nanostructured lipid carriers as a promising food grade delivery system for rutin. J Funct Foods 2016;26:167-75

9. Belfrage P, Vaughan M. Simple liquid-liquid partition system for isolation of labeled oleic acid from mixtures with glycerides. J Lipid Res 1969;10:341-4.

10. Bouwmeester H, Dekkers S, Noordam MY, Hagens WI, Bulder AS, de Heer C, et al. Review of health safety aspects of nanotechnologies in food production. Regul Toxicol Pharmacol 2009;53:52-62.

11. Ali H, El-Sayed K, Sylvester PW, Nazzal S. Molecular interaction and localization of tocotrienol-rich fraction (TRF) within the matrices of lipid nanoparticles: Evidence studies by differential scanning calorimetry (DSC) and proton nuclear magnetic resonance spectroscopy ((1)H NMR). Colloids Surf B Biointerfaces 2010;77:286-97.

12. Chen H, Chang X, Du D, Liu W, Liu J, Weng T, et al. Podophyllotoxinloaded solid lipid nanoparticles for epidermal targeting. J Control Release 2006;110:296-306

13. Cui Z, Mumper RJ. Genetic immunization using nanoparticles engineered from microemulsion precursors. Pharm Res 2002;19:939-46. 
14. Mishra B, Patel BB, Tiwari S. Colloidal nanocarriers: A review on formulation technology, types and applications toward targeted drug delivery. Nanomedicine 2010;6:9-24

15. Müller RH, Maassen S, Weyhers H, Mehnert W. Phagocytic uptake and cytotoxicity of solid lipid nanoparticles (SLN) sterically stabilized with poloxamine 908 and poloxamer 407. J Drug Target 1996;4:161-70.

16. Puglia C, Blasi P, Rizza L, Schoubben A, Bonina F, Rossi C, et al. Lipid nanoparticles for prolonged topical delivery: An in vitro and in vivo investigation. Int J Pharm 2008;357:295-304.

17. Patidar A, Thakur DS, Kumar P, Verma J. A review on novel lipid based carrier. Int J Pharm Pharm Sci 2010;2:30-5.

18. R.H. Müller and J.S. Lucks, European Patent 0605497 (1996).

19. Müller RH, Runge SA, Ravelli V. German Patent Application DE 19819273 A1; 1998.

20. Penkler L, Müller RH, Runge SA, Ravelli V. Extended Patent on the Basis of (2), PCT Application PCT/EP99/02892; 1999.

21. M.R. Gasco, Patent 5250236 (1993)

22. Boltri L, Canal T, Esposito PA, Carli F. Lipid nanoparticles: Evaluation of some critical formulation parameters. Proc Int Symp Control Rel Bioact Mater 1993;20:346-7.

23. Carmona-Ribeiro AM. Biomimetic nanoparticles: Preparation, characterization and biomedical applications. Int $\mathrm{J}$ Nanomedicine 2010;5:249-59.

24. Sawant KK, Dodiya SS. Recent advances and patents on solid lipid nanoparticles. Recent Pat Drug Deliv Formul 2008;2:120-35.

25. Müller RH, Mäder K, Gohla S. Solid lipid nanoparticles (SLN) for controlled drug delivery - A review of the state of the art. Eur J Pharm Biopharm 2000;50:161-77.

26. Jores K, Mehnert W, Mäder K. Physicochemical investigations on solid lipid nanoparticles and on oil-loaded solid lipid nanoparticles: A nuclear magnetic resonance and electron spin resonance study. Pharm Res 2003;20:1274-83.

27. Shidhaye SS, Vaidya R, Sutar S, Patwardhan A, Kadam VJ. Solid lipid nanoparticles and nanostructured lipid carriers - innovative generations of solid lipid carriers. Curr Drug Deliv 2008;5:324-31.

28. Lancelot A, Sierra T, Serrano JL. Nanostructured liquid-crystalline particles for drug delivery. Expert Opin Drug Deliv 2014;11:547-64

29. Pardeike J, Hommoss A, Müller RH. Lipid nanoparticles (SLN, NLC) in cosmetic and pharmaceutical dermal products. Int J Pharm 2009;366:170-84.

30. Liu $\mathrm{C} \mathrm{H}, \mathrm{Wu} \mathrm{CT}$. Optimization of nanostructured lipid carriers for lutein delivery. Colloid Surf A 2010;353:149-56.

31. Mk Sahu, Gc Soni, et al International Journal For Pharmaceutical Research Scholars (Ijprs), 2012, 1(3)

32. Cavalli R, Gasco MR, Chetoni P, Burgalassi S, Saettone MF. Solid lipid nanoparticles (SLN) as ocular delivery system for tobramycin. Int J Pharm 2002;238:241-5.

33. Chattopadhyay P, Shekunov BY, Yim D, Cipolla D, Boyd B, Farr S, et al. Production of solid lipid nanoparticle suspensions using supercritical fluid extraction of emulsions (SFEE) for pulmonary delivery using the AERx system. Adv Drug Deliv Rev 2007;59:444-53.

34. Manjunath K, Venkateswarlu V. Pharmacokinetics, tissue distribution and bioavailability of clozapine solid lipid nanoparticles after intravenous and intraduodenal administration. J Control Release 2005; 107:215-28.

35. Sivaramakrishnan R, Nakamura C, Mehnert W, Korting HC, Kramer KD, Schäfer-Korting M. Glucocorticoid entrapment into lipid carriers - characterisation by parelectric spectroscopy and influence on dermal uptake. J Control Release 2004;97:493-502.

36. Puri A, Loomis K, Smith B, Lee JH, Yavlovich A, Heldman E, et al. Lipid-based nanoparticles as pharmaceutical drug carriers: From concepts to clinic. Crit Rev Ther Drug Carrier Syst 2009;26:523-80.

37. Gregoriadis G, Florence AT, Patel HM. Liposomes in drug delivery. In: Florence AT, Chur GG, editors. Drug Targeting and Delivery. Chur: Harwood Academic Publishers GmbH; 1993.

38. Choi MJ, Maibach HI. Liposomes and niosomes as topical drug delivery systems. Skin Pharmacol Physiol 2005;18:209-19.

39. Subramanian S, Anandam S, Krishnamoorthy K, Rajappan M. Nanosponges: A novel class of drug delivery system - review. J Pharm Pharm Sci 2012;15:103-11.

40. Charcosset C,El-HaratiA, Fessi H. Preparation of solid lipid nanoparticles using a membrane contactor. J Control Release 2005;108:112-20.

41. Chaudhry Q, Castle L. Food applications of nanotechnologies: An overview of opportunities and challenges for developing countries. Trends Food Sci Technol 2011;22:595-603.

42. Chaudhry Q, Scotter M, Blackburn J, Ross B, Boxall A, Castle L, et al. Applications and implications of nanotechnologies for the food sector.
Food Addit Contam Part A Chem Anal Control Expo Risk Assess 2008;25:241-58.

43. Chaudhry Q, Castle L, Watkins R. Nanotechnologies in Food. London: Royal Society of Chemistry; 2010.

44. Chawla V, Saraf SA. Glyceryl behenate and its suitability for production of aceclofenac solid lipid nanoparticles. J Am Oil Chem Soc 2011;88:119-26.

45. Garcia-Fuentes M, Alonso MJ, Torres D. Design and characterization of a new drug nanocarrier made from solid-liquid lipid mixtures. J Colloid Interface Sci 2005;285:590-8.

46. González-Mira E, Nikolić S, García ML, Egea MA, Souto EB, Calpena AC, et al. Potential use of nanostructured lipid carriers for topical delivery of flurbiprofen. J Pharm Sci 2011;100:242-51.

47. Han F, Li S, Yin R, Liu H, Xu L. Effect of surfactants on the formation and characterization of a new type of colloidal drug delivery system: Nanostructured lipid carriers. Colloids Surf A Physicochem Eng Aspects 2008;315:210-6.

48. Higuchi WI. Analysis of data on the medication release from ointments. J Pharm Sci 1962;51:802-4.

49. Jackson CL, McKenna GB. The melting behavior of organic materials confined in porous solids. J Chem Phys 1990;93:9002-11.

50. Mukherjee S, Ray S, Thakur RS. Solid lipid nanoparticles: A modern formulation approach in drug delivery system. Indian J Pharm Sci 2009;71:349-58.

51. Zauner W, Farrow NA, Haines AM. In vitro uptake of polystyrene microspheres: Effect of particle size, cell line and cell density. J Control Release 2001;71:39-51.

52. Müller RH, Rühl D, Runge S, Schulze-Forster K, Mehnert W. Cytotoxicity of solid lipid nanoparticles as a function of the lipid matrix and the surfactant. Pharm Res 1997;14:458-62.

53. Radtke M, Muller RH. Nanostructured lipid carriers: The new generation of lipid drug carriers. New Drugs 2001;2:48-52.

54. Saupe A, Wissing SA, Lenk A, Schmidt C, Müller RH. Solid lipid nanoparticles (SLN) and nanostructured lipid carriers (NLC) Structural investigations on two different carrier systems. Biomed Mater Eng 2005;15:393-402.

55. Müller RH, Radtke M, Wissing SA. Solid lipid nanoparticles (SLN) and nanostructured lipid carriers (NLC) in cosmetic and dermatological preparations. Adv Drug Deliv Rev 2002;54 Suppl 1:S131-55.

56. Jenning V, Schäfer-Korting M, Gohla S. Vitamin A-loaded solid lipid nanoparticles for topical use: Drug release properties. J Control Release 2000;66:115-26

57. Tsai MJ, Wu PC, Huang YB, Chang JS, Lin CL, Tsai YH, et al. Baicalein loaded in tocol nanostructured lipid carriers (tocol NLCs) for enhanced stability and brain targeting. Int J Pharm 2012;423:461-70.

58. Souto EB, Müller RH. Cosmetic features and applications of lipid nanoparticles (SLN, NLC). Int J Cosmet Sci 2008;30:157-65.

59. Pokharkar VB, Jolly MR, Kumbhar DD. Engineering of a hybrid polymer-lipid nanocarrier for the nasal delivery of tenofovir disoproxil fumarate: Physicochemical, molecular, microstructural, and stability evaluation. Eur J Pharm Sci 2015;71:99-111.

60. Mishra BJ, Kaul A, Trivedi P. L-cysteine conjugated poly L-lactide nanoparticles containing 5-fluorouracil: Formulation, characterization, release and uptake by tissues in vivo. Drug Deliv 2015;22:214-22.

61. Araújo J, Gonzalez E, Egea MA, Garcia ML, Souto EB. Nanomedicines for ocular NSAIDs: Safety on drug delivery. Nanomedicine 2009;5:394-401.

62. Müller RH, Souto EB, Radtke M. PCT Application PCT/EP00/04111; 2000.

63. Rosenblatt KM, Bunjes H. Poly(vinyl alcohol) as emulsifier stabilizes solid triglyceride drug carrier nanoparticles in the $\alpha$-modification. Mol Pharm 2009;6:105-20.

64. Kakar S, Batra D, Singh R. Preparation and evaluation of magnetic microspheres of mesalamine for colon drug delivery. J Acute Dis 2013;2:226-31

65. Obeidat WM, Schwabe K, Müller RH, Keck CM. Preservation of nanostructured lipid carriers (NLC). Eur J Pharm Biopharm 2010;76:56-67.

66. Oldrich C, Bakowski U, Lehr CM, Müller RH, Kneuer C. Cationic solid- lipid nanoparticles can efficiently bind and transfect plasmid DNA. J Control Release 2001;77:345-55.

67. Acener M. Preparation, characterization and physicochemical properties of solid lipid nanoparticles (SLN) and nanostructured lipid carriers (NLC): Their benefits as colloidal drug carrier systems. Pharmazie 2006;61:375.

68. Shekhawat PB. Preparation and evaluation of clotrimazole nanostructured lipid carrier for topical delivery. Int J Pharm Bio Sci 2013;4:407-16. 